\title{
Presenting an Effective Method to Detect and Track the Broken Path in VANET Using UAVs
}

\author{
Zohreh Bakhtiari $\left(\mathbb{D},{ }^{1}\right.$ Rozita Jamili Oskouei $\mathbb{D}^{1},{ }^{1}$ Mona Soleymani ${ }^{(\mathbb{D}}{ }^{2}$ \\ and Akhtar Hussain Jalbani ${ }^{3}{ }^{3}$ \\ ${ }^{1}$ Department of Computer Science and Information Technology, Mahdishahr Branch, Islamic Azad University, Mahdishahr, Iran \\ ${ }^{2}$ Department of Computer Engineering, Parand Branch, Islamic Azad University, Tehran, Iran \\ ${ }^{3}$ IT Department, QUAID-E-AWAM University of Engineering, Science \& Technology, Nawabshah, Sindh, Pakistan
}

Correspondence should be addressed to Rozita Jamili Oskouei; rozita2010r@gmail.com

Received 19 April 2020; Revised 21 May 2020; Accepted 10 June 2020; Published 17 August 2020

Academic Editor: Fawad Zaman

Copyright (c) 2020 Zohreh Bakhtiari et al. This is an open access article distributed under the Creative Commons Attribution License, which permits unrestricted use, distribution, and reproduction in any medium, provided the original work is properly cited.

\begin{abstract}
The routing process in vehicular ad hoc networks (VANETs) is a challenging task in urban areas which is due to the high mobility of vehicles, repetitive defects of the communication path, and the various barriers that may affect the reliability of data transmission and routing. Accordingly, the connectivity in vehicular communications has received the researchers' attention, so different geographic routing protocols have been proposed in this respect. Unmanned aerial vehicles (UAVs) are useful for overcoming routing constraints. Cloud computing has also been defined as a new infrastructure for VANET which is made up of a significant number of computing nodes including stable data centers as well as a set of mobile computing devices embedded on vehicles. The aim of this research is to simulate a VANET in an urban area using cloud computing infrastructure and applying unmanned aerial vehicles (UAV) so that the negative influence of barriers in packet delivery and routing is avoided. To evaluate, the proposed method is compared with the basic protocol ClouDiV. Ns-2 simulation results show that the proposed method outperforms with different densities and variable times in terms of efficiency and performance.
\end{abstract}

\section{Introduction}

Vehicular ad hoc network (VANET) is a kind of Ad hoc networks that provide the communication among the adjacent vehicles, as well as the fixed equipment and vehicles that are usually installed along the roads. The analysis of connections in these networks is important in a way that in VANET, due to the high mobility over other ad hoc networks and frequent changes in the network topology, the path break may occur frequently. The previous researches are more about the relationship between the vehicles' connectivity and distance which have been studied in terms of the speed effect, vehicle density, different mobility patterns, radio broadcast range, node rank, connection duration, etc. All of these analyses are effective in the selection of the next hop in routing in order to prevent the path breakage [1]. The routing protocols for VANETs are the main issue of this technology due to the high dynamic nature of the nodes.
Since the effective and reliable dissemination is a major challenge in VANET, the geographical routing protocols are flexible for most ITS applications. The characteristics of urban traffic environment differentiate it from the highway traffic area [2]. The weather conditions also have a significant impact on this traffic environment. In urban spaces, the buildings have blocked the direct communication between two vehicles as barriers. This prevents vehicles from exchanging messages even when they are within the same transmission range. The ClouDiV protocol has been proposed as a geographic protocol for message dissemination in vehicular networks based on the cloud computing infrastructure which has recently been implemented in the field of cloud computing to deal with vehicular network issues [3]. The broken paths in VANET will be caused due to the loss of connection or the presence of network gaps and by the use of UAVs or unmanned aerial vehicles (also referred to as remotely controlled bird objects) in the communication systems can 
detect these paths and share the connection information among vehicles.

In this paper, the proposed scheme is based on the Clou$\mathrm{DiV}$ protocol. In the proposed method, by making changes in the structure of the ClouDiV algorithm, we hope that this modified protocol accompanied with the UAVs in the sky improves the routing performance even with the presence of barriers. The proposed method is evaluated and compared to detect and trace the broken communication paths in VANETs based on the ClouDiV protocol. The evaluations are performed in terms of some criteria such as packet delivery rate, end-toend latency, throughput, and the number of lost packets.

The organization of this paper is as follows: In Section 2, the background and related works are given. In Section 3, the details of the proposed method are described with the given goals. Section 4 presents the evaluation of the proposed method by analyzing the information and giving the simulation results. Finally, Section 5 gives the conclusion and future works.

\section{Related Works}

By introducing VANETs and increasing the capabilities of VANET, the consumer applications have been enabled, and the subject of connection analysis has attracted more attention from research communities [4]-[9]. In the following sections, while studying the connection analysis in the VANETs, we are going to discuss the recent works on cloud computing and UAV performance in communications and interconnections of these networks.

\subsection{Analysis of Connections and Communication in VANET.} Recently, connectivity has attracted the attention of researchers in vehicular communications, and various routing protocols have been proposed in this field. To reflect the high dynamics of the connection due to different patterns of mobility, the effects of speed $[4,6,9]$, vehicle density [4, $10]$, radio broadcasting range $[6,9,10]$, node rank [11], and connection duration [12] have been studied.

In [13], Wu has modeled the probability of accurately connecting two nodes with distance $L$ over a linear network by the Poisson point process. In [14], the authors have studied the data dissemination approaches for highway scenarios in vehicular networks using roadside units in order to achieve a communicative coverage among vehicles that can be extended at some levels of their connection where this method is also useful for critical time data dissemination. In [15], a modeling method has been studied in uplink v2v communications for highways and rural, urban, and suburban environments [15]. To analyze the interconnection of wireless vehicular networks, the authors in [16] have examined the maximum number of hops in a multiple-hop path from the source to the destination and the minimum transmission power used to ensure the connection of the vehicles from the physical layer's viewpoint. The test modeling of the vehicle-to-vehicle lost path in urban, suburban, and highways at $5.8 \mathrm{GHz}$ [17] has been carried out by the definition of free space, where the results are similar to the previous values. In [1], Zarei et al. has presented a scheme to analyze the connection for the dynamic motion of wireless vehicular networks that accurately calculated the connection distance for a one-way highway path and proposed a new formula for the time-dependent probability density function (pdf). Also, an analysis of the v2v connection for wireless vehicular networks has been done using mathematical models [18]. In [19], the authors have presented a combined HRAR routeaware routing protocol in urban and highway environments based on a two-level hierarchical routing in terms of road communications in VANETs. An iCAR-ll infrastructurebased and connection aware routing protocol has been proposed in [20]. This protocol consists of a number of algorithms to be run by vehicles, and it has been applied to predict the local network connection and update local servers with real-time network information in order to build a global network topology. In [21], the authors have proposed the Fast MF to extend the Internet access for vehicular nodes and get access to these nodes from any remote server for the connected vehicles under information-based architectures. A cluster-based file transfer scheme for Highway VANETs (CFT) has been presented in [22]. With CFT, when the requested file cannot be successfully transmitted from the source to the destination, a cluster is created under a direct $\mathrm{v} 2 \mathrm{v}$ connection and the file is transmitted in several hops. In these researches, three models have been confirmed to study the problem of file transfer in VANETs: (1) high mobility of vehicles, (2) connection time prediction, and (3) v2v communications. In [23], in order to disseminate the connection aware information by estimating the ability of node transmission in nearly connected VANETs (CADDs), first, the connection specifications are examined theoretically and then CADD is presented to improve data dissemination. A connection aware routing protocol of the road network has been presented for wireless vehicular networks in [24]. The protocol tries to avoid the clustering phenomenon under the influence of traffic lights and to select the road section by disseminating information among more vehicles. Li et al. [25] have presented multihop transmission latency and connection probability in the analysis of multihop links from the $5 \mathrm{G}$ enabled vehicular networks. In [26], the authors have proposed a reliable IP-based routing in VAENT with network gaps based on road segments and virtual nodes that can form routing paths without network gaps (the network gap occurs when the distance between two nonadjacent vehicles is more than the transmission radius).

For vehicular communications in $700 \mathrm{MHz}$ and $5.9 \mathrm{GHz}$, under the LOS and NLOS conditions, a simple dissemination model suitable for VAENT simulations has been proposed to evaluate the protocol and system setting in [27], where the lost path has been detected by narrow channel measurement. Liu et al. [28] have performed the path-loss modeling for $\mathrm{v} 2 \mathrm{v}$ communications on an uplink path using low-height antennas at downhill and uphill in different scenarios. In [29], the authors have presented the next hop selection scheme based on the remaining lifetime of the link for wireless vehicular networks and proposed an algorithm to predict the remaining link lifetime in VANETs using a Kalman filter based on the prediction technique. [30] have presented a stable path and decision-making scheme to send a data packet 
in a highway which allows the prediction of the path's lifetime and remaining time for data packet transmission and removes the path's failure message, since the source vehicle knows the time when the path would be broken. In this scheme, the packet delivery rate is reduced by increasing the network density which is due to this fact that yet no method has been presented to restore the lost data packets.

All these studies and analyses are effective in selecting the next hop to prevent the breakage of the communication path. Also, the strategies of path repair [26], packet broadcast verification [30], and using guards have been proposed to help the process of restoring the lost paths when the network failed [18].

\subsection{Cloud Computing in VANET Communication. Recently,} some researches have proposed cloud computing to overcome the vehicular network problems [3]. Among them, a new routing scheme, called VehiCloud [31], has been built to overcome the unreliable $\mathrm{v} 2 \mathrm{v}$ communications and it develops the constrained computing capabilities of vehicular devices by using cloud computing architecture. This method has suggested that each vehicle predicts its future successes by generating point messages which is the description of vehicle's trajectory. In [32], the integrated Internet access is another issue that has been solved by the cloud computing infrastructure. The vehicular network literature has mentioned another vehicular scheme which is based on cloud, called vehicular cloud model (VC) [33]. It has been defined as a new mobile cloud computing model. The VC simulation study shows that the increased network density leads to lower latency in dissemination. In [34], three main cloud entities are proposed: vehicular cloud (VC), vehicles using cloud $(\mathrm{VuC})$, and hybrid cloud (HC). A new approach of using RSU as a cloud server has been proposed in [35]. To provide safe and unsafe services in the vehicular applications, the vehicular cloud (VCR) has been proposed for roadside scenario in [36].

Bitam and Mellouk [3] have presented cloud computingbased message dissemination protocol for wireless vehicular networks to ensure reliable connections. ClouDiV has been considered a geographical protocol which will disseminate messages through a connection process based on cloud data centers. To evaluate the performance of this protocol, an extensive experimental study has been done in terms of average point to point latency, packet delivery rate, and normalized overhead, and the obtained results have been compared with RIVER protocol.

2.3. UAVs on VANET Connections. Recently, extensive researches have been performed in the field of routing and tracing the communication path between two vehicles in a VANET to disseminate data packets; however, most of them have not considered UAVs to solve the problem of disconnection, frequently occurring in urban areas, and only a number of them have used UAV to overcome the obstaclebased disconnection in urban areas. The authors in [37] have proposed a new mechanism in mobile ad hoc networks that is the usage of UAVs to increase the connection. The major drawback of this proposed protocol is that UAVs have not used GPS information and trajectory (path) measurement during path detection and data transmission. The traffic congestion aware routing based on connection using UAVs for VANETs (CRUV) [38] is a new routing method for VANETs that is able to detect the shortest connected path at any time to effectively send packets to their destinations by combining the real-time traffic congestion in terms of periodic exchange of Hello Messages and Dijkstra algorithm in order to determine the shortest path from the source to the destination. UVAR (VANET routing protocol by UAV) [39] is a new routing protocol in collaboration with the unmanned vehicles in sky to give a general view on the connected segments and improve the routing process. To prevent the previous limitations, UVAR protocol considers the real distribution of the vehicles in the selected parts of the road which not only provides the accurate calculation of the vehicle connection but also solves the current barriers that manipulate the calculations. Despite the use of the general rule as in [40] to send data packets in earth, UVAR has completely abused UAVs, so that the barriers in the sky would not occur anymore.

Data delivery has been optimized by using this combination of communications between the vehicles and UAVs which would help to reduce the loss of packets and delivery latency.

\section{An Effective Way to Detect and Trace the Broken Paths in VANET Using UAVs}

The main goal of this paper is to prevent the negative impact of barriers in packet delivery and routing in urban areas by using cloud computing infrastructure and UAVs and to detect these paths by using ClouDiV protocol in those parts of the road that the communicative paths are broken and to share the connection information among vehicles by using UAVs (unmanned aerial vehicles) as well as to find the broken paths so that at last the reliable and stable paths can be created. The ClouDiV protocol performs rebroadcast action for data dissemination in some cases, such as disconnection of the communication path that imposes an overhead on the network. The use of unmanned aerial vehicles for routing with ClouDiV will cause the disconnected communication paths to be identified and repaired prior to the data dissemination, and there is no need for a broadcasting operation, so the overhead of this protocol will decrease and the throughput will increase.

In this proposed scheme in some parts and structure of the ClouDiV protocol will be modified so that this protocol would have a general view on the connected parts with the UAVs in the sky and even with the presence of barriers (buildings, high trees, etc.), the routing would be improved. In this method, UAVs share the connection information for all VANET nodes and data centers and help updating the routing tables of data centers, and they are applied in the parts of the road where there is a problem of barriers to send data packets as other VANET nodes. In the proposed scheme, the packet delivery will be improved by decreasing the delivery latency and packet loss.

Nodes in VANET make traffic and motion and have high dynamicity; for this, the connection path between the source and destination nodes is frequently disconnected. In a 

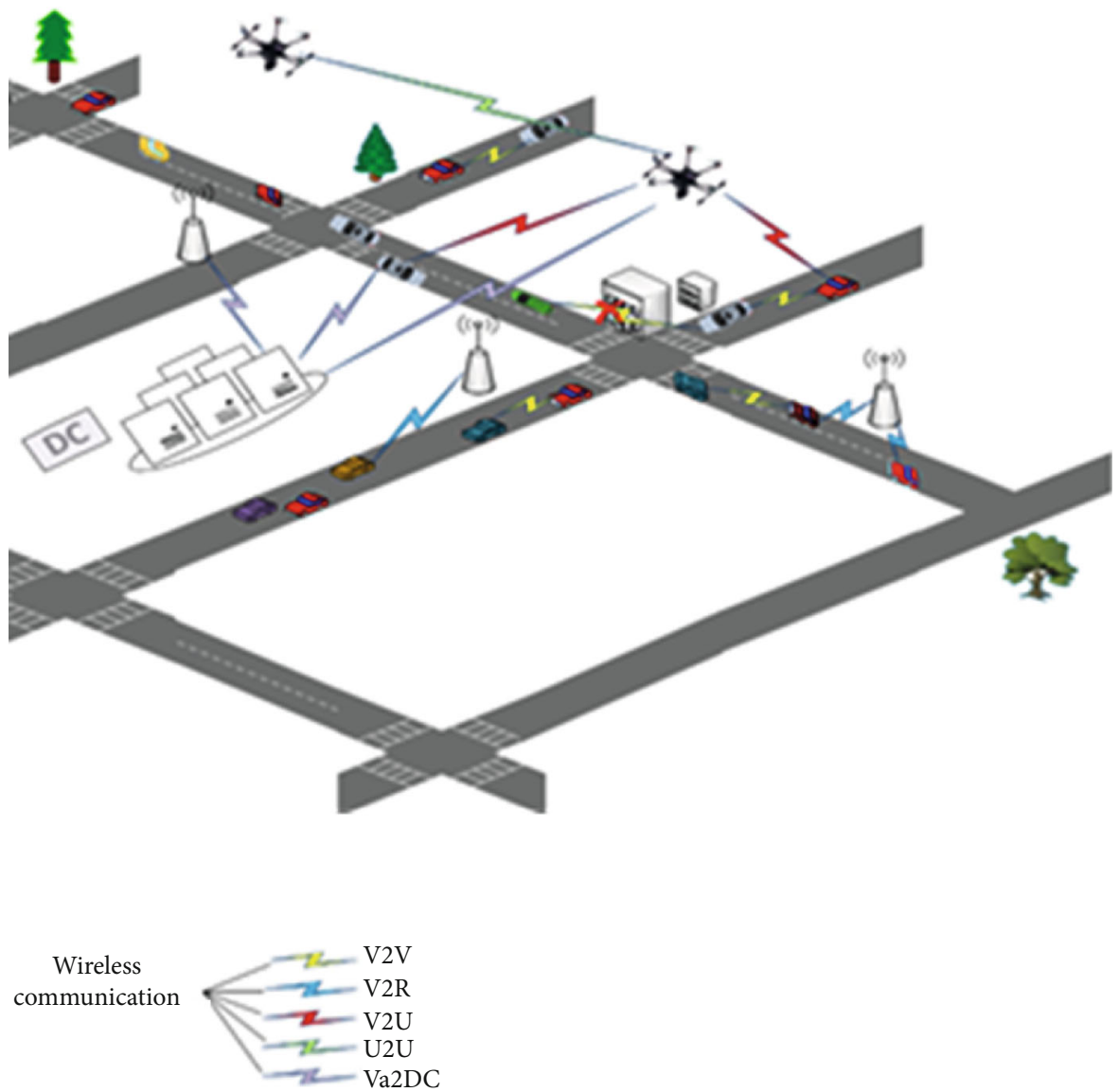

Figure 1: Vehicle and UAV communication.

VANET, the mere existence of a multihop path from source to destination cannot always ensure that linkage from source to destination is successful, since the wireless vehicular challenges are failure prone. To ensure the reliable information dissemination in a critical time, the network connection must be guaranteed. Due to the fact that in VANETs, the mobility with high speed will lead to the frequent changes in the network topology, these changes will increase the rate of frequent disconnection and packet loss during dissemination and furthermore; the packet loss is inevitable due to the network gaps. Therefore, the detection of the disconnected path in VANETs could be used to reduce the rate of packet loss and transmission delay in the geographical routing.

Due to the variable speed of moving vehicles and vehicle movements that are limited by roads and traffic lights, the main goals to be considered are maintaining the created connection, preventing the failure of the link, which happens frequently and reducing the process of disconnection and reconnection of the node through a new path to increase the network performance, reduce packet loss rates, and improve connectivity as well as reduce the loss of communication path in VANET by detecting the broken communication path in the geographical routing process. Also, the consideration of the characteristics of the urban traffic environment is aimed at reducing the negative impact of urban barriers on routing protocols. Finally, the ultimate goals for this study include reducing the road accidents and damages caused by them, improving roads and streets' safety, and improving the environmental conditions. We also hope that this project will be used and exploited by the ministry of roads and urban development and automobile companies accompanied with road transportation authority.

In order to outline the proposed method, we will first provide the architecture, and then, along with a review of the ClouDiV protocol, the changes that made to this protocol will be explained.

3.1. The Proposed System Architecture. As shown in Figure 1, this system consists of a set of vehicles, UAVs, roadside units, and data centers distributed over the network. In this hybrid communicative system, the IEEE 802.11P MAC protocol has been confirmed for $\mathrm{v} 2 \mathrm{v}$ communications, UAV-to-vehicle communications, and VANET node communications (vehicle, UAV, and RSU) with data center. Five types of wireless communications are used in this system:

Communications include vehicle to vehicle (V2V), vehicle to roadside units (V2R), vehicle to UAV (V2U), unmanned aerial vehicles to unmanned aerial vehicles (U2U) [39], and VANET node with data center (Vn2DC). Data centers are considered the fixed nodes of cloud computing, as cloud servers, and have wireless communication with VANET nodes. However, the data center communication is wired. 


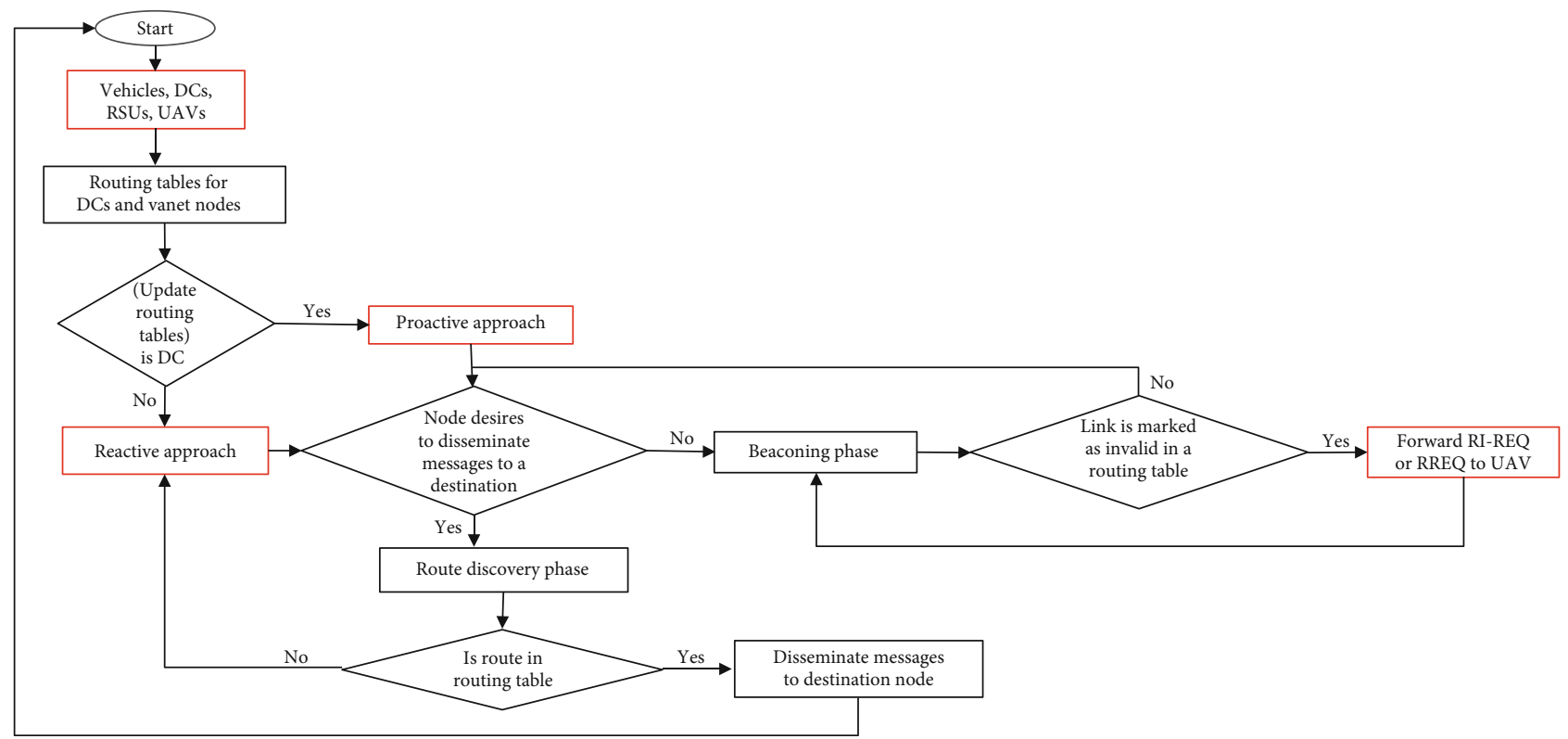

FIgURE 2: The proposed method flowchart.

3.2. Overview on ClouDiV Protocol Performance. ClouDiV [3] is a message dissemination protocol for vehicular networks based on cloud computing architecture which is considered a geographical protocol. ClouDiV has combined a fixed cloud computing infrastructure (e.g., data centers) and a flexible cloud structure based on computers embedded on the vehicles.

In this protocol, it is assumed that the vehicular network consists of a number of different VANET nodes (e.g., vehicles and roadside units); also, different cloud servers such as data centers are placed in the traffic environment. ClouDiV is considered a hybrid message dissemination protocol where a proactive approach is applied by each data center in order to detect new and updated paths of each node. In addition, a reactive approach is defined to be performed by each vehicle with the aim of finding the nearest data center as an intermediate node. Also, two types of routing tables are proposed: data center routing table and VANET node routing table. Each points out to using a particular type of a routing process. Beaconing phase with the aim of ensuring the routing tables' updating by Beacon packets transmitted periodically. In path detection phase, to detect a path, two complement processes are applied: proactive routing and reactive routing.

3.3. The Proposed Method. Some parts of the ClouDiV protocol have been modified to reach the given goals (Figure 2). The data center can send its request to the UAVs at the transfer range to update its routing table in the proactive routing when the control packet of requesting the path information (RI-REQ) with the aim of collecting different information of routing reaches to a node that could not respond to its requested information; in other words, it cannot find a node in its neighboring area over the earth. Since in this protocol, the routing information among VANET nodes can be stored and updated without cloud computing structure (beaconing phase); in this method, the VANET node can collect infor- mation from the neighbor nodes to prevent recording invalid connections and to cause packet failure by using UAVs. In other words, if it cannot receive the information from its neighbor node in a given time period, it can perform this action by UAVs. Also, in this scheme, in the phase of detecting a path in the reactive routing, the RREQ packet can be sent to the UAV placed within the given range instead of broadcasting the route request control packet (RREQ) by the source node which helps to significantly reduce the routing overhead.

The routing tables of the proposed method are the same as the ClouDiV protocol as well as the data center routing table and VANET node routing table. The ClouDiV routing process will be performed in the path detection phase which will be performed with slight changes in the proposed method.

3.3.1. Proactive Approach. The proactive routing process in this approach is performed by each data center in the network with the aim of tracing all paths from this data center toward each node in the network (known as a future destination) [3]. If the node does not receive the information from the neighbor nodes (VANET nodes on the earth) in a given time interval, a process as beaconing phase (Figure 3 ) is performed in the broken path detection and broadcasting it by $\mathrm{UAV}$, and then, each node receiving RI-REQ generates a RI-REP so that to make communication, it sends its updated routing information to DC1. After receiving all RI-REPs, DC updates it routing table and directs the input paths toward different destinations.

3.3.2. Reactive Approach. The reactive approach is performed by each vehicle and UAV with the aim of finding the nearest data center as an intermediate node; each one tries to provide a new path for the given destination. In the reactive routing process, a path request packet (RREQ) is generated based 


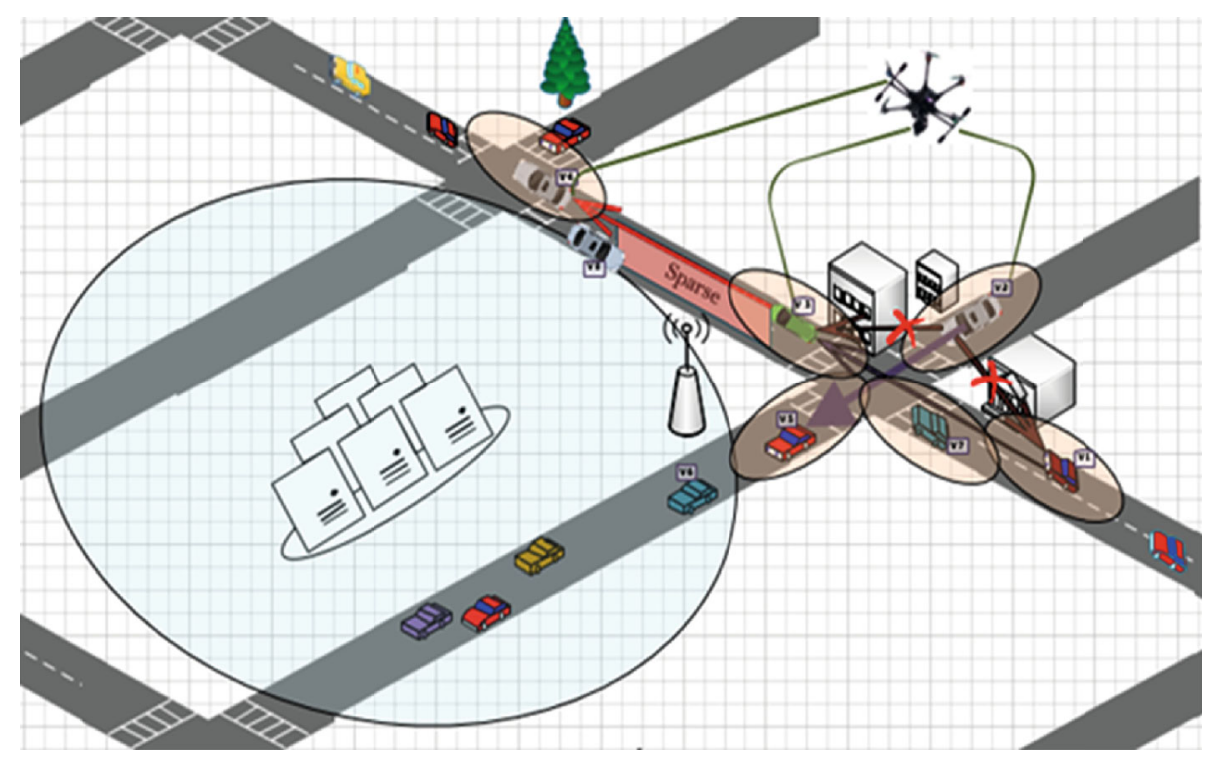

Figure 3: Disconnection of vehicles.

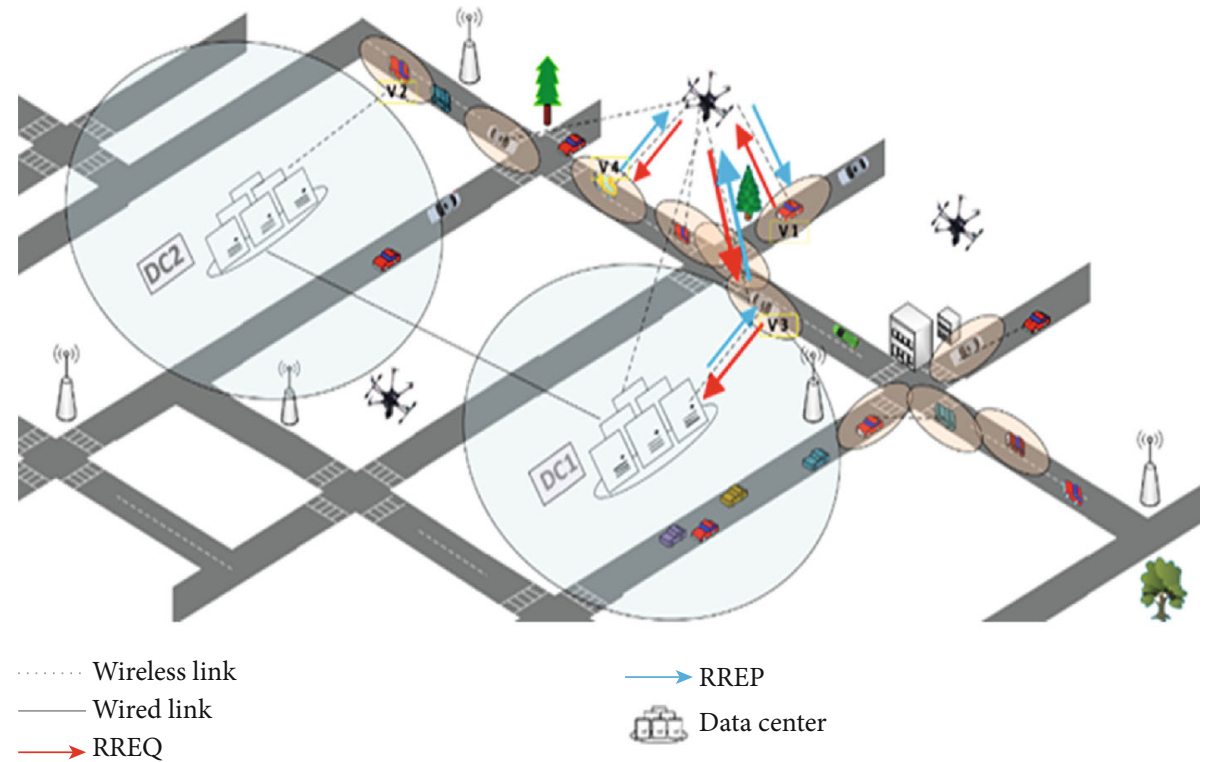

FIgURE 4: The reactive routing process of the proposed scheme.

on the request of the source node $\mathrm{V} 1$ to disseminate messages to a destination node V2 (Figure 4). In the proposed method, the RREQ is sent to the UAV within the transmission range by the source node instead of randomly broadcasted by the source node among the neighbors at that moment. The UAV node checks its routing table, and if there is a data center in its routing table, then it generates a routing response packet (RREP) and sends it to the source node; otherwise, the RREQ packet is broadcasted by UAV to the neighbor nodes within its range randomly. Here, it is assumed that the vehicles V3 and V4 have received the RREQ packet. The first data center, like DC1, has been detected by V3. As the packet has received to DC1, this data center generates a RREP and sends it to V1 across the opposite direction; there- fore, the response packet will be sent to the source node along the same path by the generated node. Similarly, the RREP packet records the next hop's node in the routing table of each visited node so that the sender represents it until it reaches the source node. As a result, the source node can begin to disseminate data packets to detect the data center through the next hop's node, which has been found by the reactive routing process. Then, the $\mathrm{DC} 1$ data center will send these data packets to the final destination, the V2 node from (DC1-DC2-V2) path detected by the last proactive routing process.

3.3.3. Beaconing Phase. In this step, the active connections between each VANET node and data center will be updated 


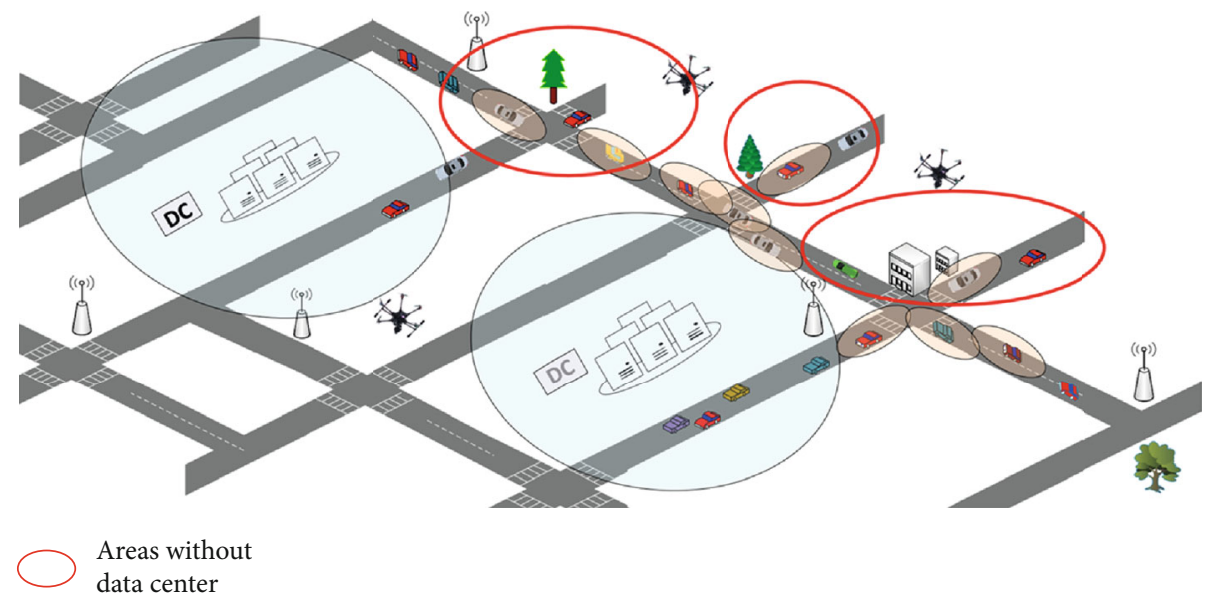

FIGURE 5: Updating routing information among VANET nodes.

by loading and updating different transmission parameters, such as measured available bandwidth and end-to-end latency. Also, the routing information among VANET nodes will also be stored and updated to perform a common routing (e.g., without the help of the cloud computing structure) in the absence of data centers [3] (Figure 5). below.

The main function of beaconing phase is described

(1) Detecting the Broken Paths. During an update, if an invalid connection is marked on a routing table, that is, one node does not receive information from neighboring nodes (VANET nodes on Earth) in a given time internal, instead of broadcasting an unavailable connection by other nodes using the error package, in the proposed scheme, according to the scenario shown in Figure 3, there is less vehicular density and the network is sparse (V3 and V4); and also, there are high buildings, which break the connection between V2 and V1, as well as V2 and V3; in such cases the earth nodes (e.g., V2) will send the path delivery information to the UAV node within the given range. The UAV node first sends the connection information to the given node in the packet response format according to its routing table, and then, the UAV will send the path error packet to the nearest data center (DC) in order to inform the data center about disconnection, so that finally, the data center will be able to replace the new paths in that range by updating its routing table. Therefore, if a request is sent to the $\mathrm{UAV}$, it indicates a disconnection that should be solved by replacing the new route.

\section{Evaluation}

To evaluate the proposed method, its overall structure is presented to simulate and examine the parameters and related criteria as follows:

This scheme consists of a cloud computing infrastructure for the VANET, including a set of fixed computing nodes such as data centers and flexible computing nodes, computers embedded on vehicles, and UAVs. In general, in this method, it is assumed that the vehicular networks are located in the traffic environment by different VANET nodes (such as vehicles, roadside units, and UAVs), as well as different cloud servers (e.g., data centers). To evaluate this scheme, network simulators such as ns2 and greencloud are used on urban scenarios.

To simulate this system and parameter settings, using a modified ClouDiV protocol, a moving pattern represents the region of an assumptive $1 * 1 \mathrm{~km}^{2}$ city with several intersections (Figure 6). In the studied network, two scenarios are considered; in both scenarios, there are a number of nodes moving between 20 and 160 where the vehicles are moving at speeds of 1 to $50 \mathrm{~km} / \mathrm{h}$. Also, a number of RSUs are distributed uniformly and a data center is used in the center of the studied area with a $1000 \mathrm{~m}$ transmission range. According to IEEE 802.11p standard, wireless accessibility in the vehicular space has been proposed for VANETs. Each vehicle has a long Wi-Fi interface of about $250 \mathrm{~m}$ to communicate with other vehicles over the same street. However, there cannot be any communication among the vehicles located on different streets due to the presence of barriers. These scenarios are simulated at variable times of 200 to $1000 \mathrm{~ms}$. The traffic flow in this simulation is CBR (UDP), and only in one of these two scenarios, a number of UAVs with $1000 \mathrm{~m}$ communication range and 40 to $90 \mathrm{~km} / \mathrm{h}$ rate are considered. The first scenario is simulated without UAVs and with Clou$\mathrm{DiV}$ protocol, and the second scenario that is the proposed scheme of this article is simulated with UAVs and the modified ClouDiV protocol, in which despite the disconnection caused by barriers and sparsity in some parts of the road, in both scenarios, to evaluate the performance of the proposed scheme, the parameters of the package delivery rate, end-to-end latency, throughput, and the number of the lost packets can be compared.

4.1. Evaluation Criteria. Four criteria are considered in the evaluation process: 


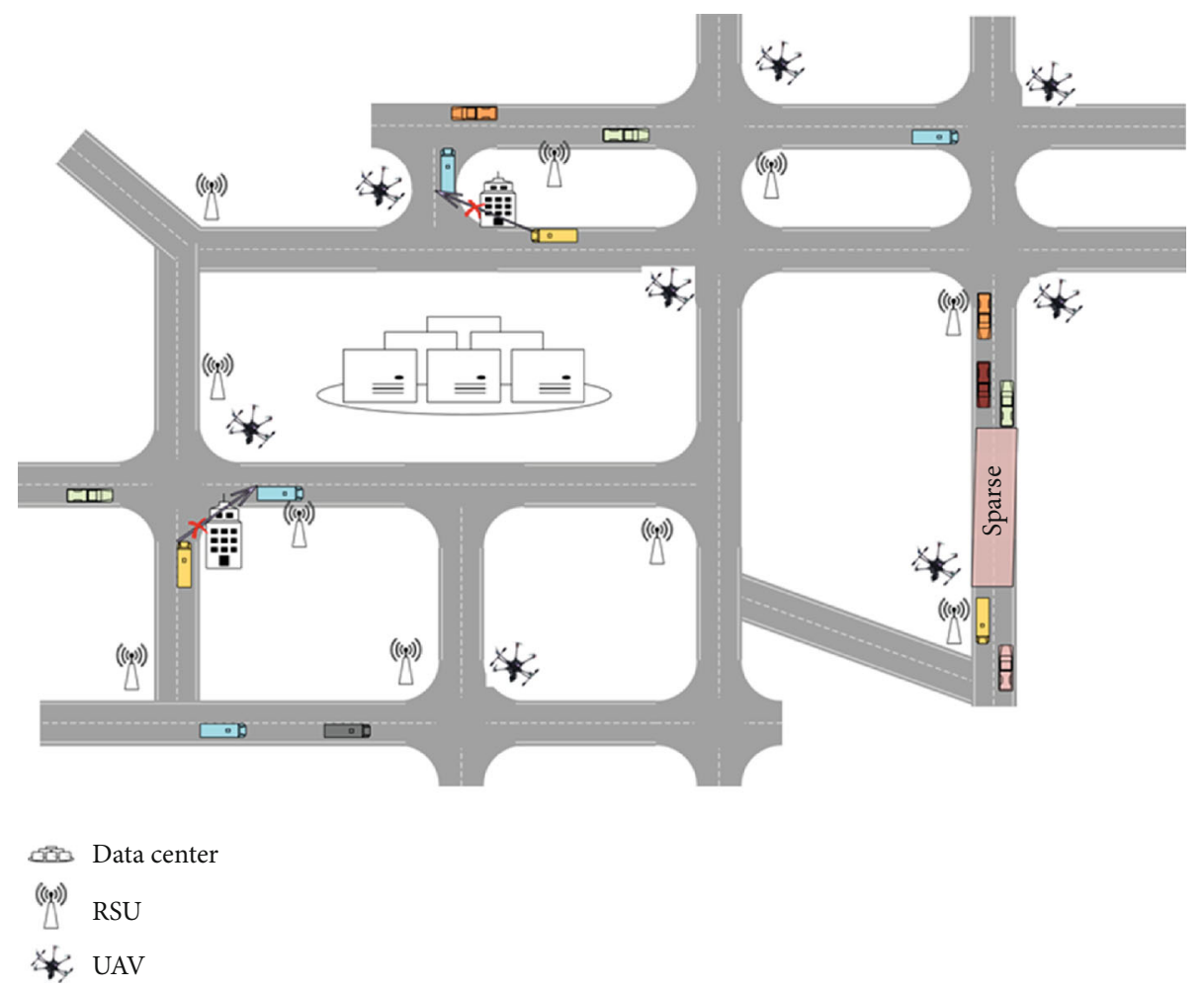

FIGURE 6: District map of the assumptive city.

Package delivery ratio: in VANETs, packets are usually sent hop-by-hop or via multiple hops. The delivery ratio of such network is calculated by

$$
\mathrm{PDR}=\left(\frac{\text { Number of sent packet }}{\text { Number of received packet }}\right) * 100 \text {. }
$$

Lost packets: packet loss in VANET networks will occur for various reasons; for example, the sent packets in the network fail to reach the destination node, or they will be lost due to a bit error or hardware failures. The presence of noise in the network can also cause the loss of packets. The loss of a number of packets in the mobile ad hoc networks can be caused by the attacks existing in such networks. This parameter usually is represented by percentage:

$$
\text { Lost packets }=\frac{(\text { Sent packet }- \text { Received packet })}{\text { Sent packet }} \text {. }
$$

According to (2), the number of the lost packets can be obtained in the VANET network.

End-to-end latency: in VANETs, a time interval in which information packets are transmitted over the network from the source node to the destination node is called an end-toend latency. This latency ends up as long as packets are transmitted from the source node to the destination node throughout the network. This parameter is represented in milliseconds.
Throughput: the reason for measuring throughput in networks is that people often tend to know the maximum data throughput in a communication link or network access. The common method for measuring this parameter is to transfer a large file from one system to another and calculate the required time to complete file transmission or copy. Then, the throughput will be achieved by dividing the file size at that time, in Mbit/s, Kbit/s, or bytes per second. The following equation calculates the throughput in a network:

$$
X=\frac{C}{T}
$$

In (3), $X$ represents the throughput, $C$ represents the number of requests completed by the system, and $T$ is the total time in which the system has been monitored.

4.2. Simulation Result Analysis. In this section, we will study and compare the data and information obtained from the proposed scheme and the ClouDiV protocol, which are based on the cloud computing structure. These data have been obtained based on the simulation by the NS-2 simulator, which is the best simulator for ad hoc networks for its flexibility and software efficiency, and it is considered a very powerful simulator for wireless vehicular networks.

At each simulation, one can change a number of parameters, such as simulation time, and evaluate the simulation results. Initially, evaluations are performed under various vehicle densities, with vehicle densities between 20 and 160 where UAV has also been taken into account as a VANET 


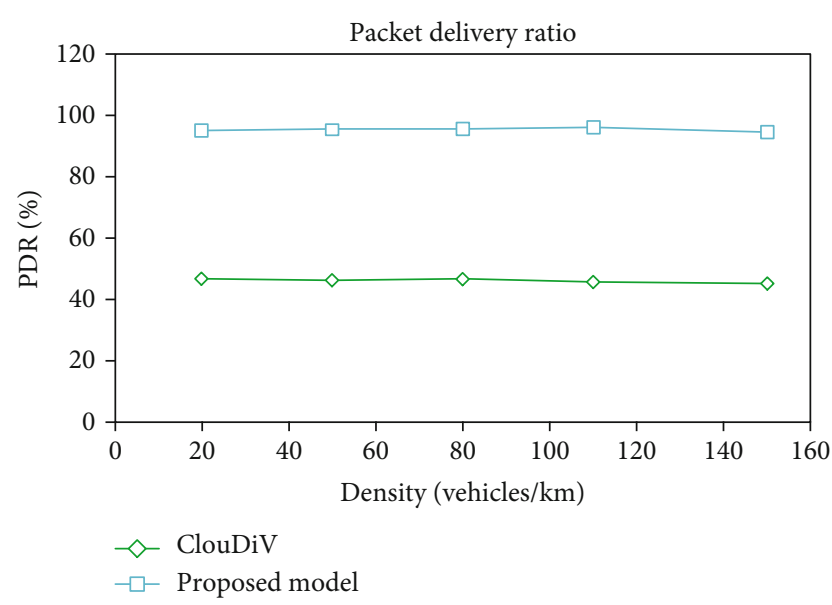

Figure 7: Packet delivery ratio with different densities.

node but with three-dimensional coordinates due to its height. Then, we have performed the tests and evaluations through simulations at different times, where we have considered the time intervals between 200 and 1000 milliseconds. The simulation results are presented as follows:

(i) Packet delivery ratio: in this section, we compare the packet delivery ratio under different vehicle densities and at variable times for the proposed method and the evaluated ClouDiV protocol. As shown in Figure 7, the simulation results show that the delivery ratio in the proposed method is higher than ClouDiV which is due to the usage of paths with ultrabandwidth provided by cloud-based infrastructures.

Also, according to Figure 8, it can be seen that the proposed model has a better performance in terms of a packet delivery ratio at different times. However, ClouDiV only uses a broadcasting method to find the route to the destination node, which will be time-consuming, and the packet delivery rate will be lower than the proposed method.

(ii) End-to-end latency: Figure 9 shows that the end-toend latency of different vehicle densities is lower for the proposed method compared to the evaluated protocol. This is due to the use of UAVs in the range, which has led to the selection of reliable paths. Therefore, short distances and optimal paths through which data packets are transmitted to the destination significantly reduce the end-to-end latency while ClouDiV adds additional time when buffering the packet according to the path detection process before delivering the packet to the destination.

And, according to Figure 10, the end-to-end latency at variable times for the proposed model is better than the Clou$\mathrm{DiV}$ protocol. In the proposed scheme, the packets are delivered more quickly, since UAVs occasionally choose a shorter distance to deliver the packet to the destination, while in ClouDiV, it is possible that the requested path cannot be detected by the source node in the path detection process.

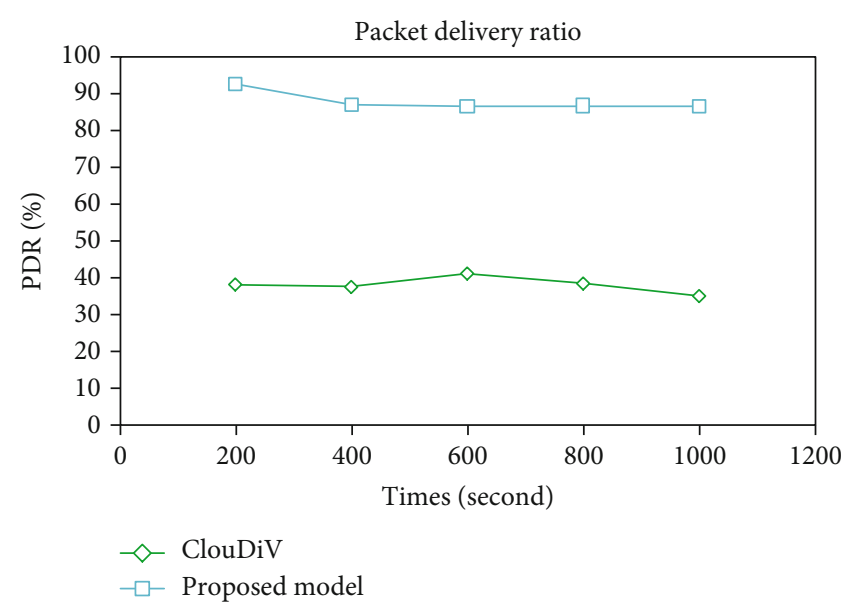

FIGURE 8: Packet delivery ratio at variable times.

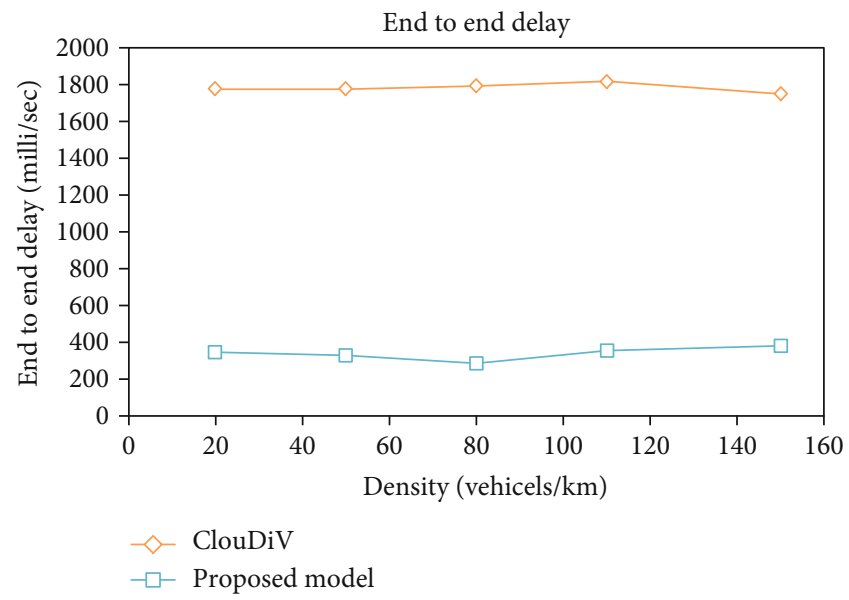

Figure 9: End-to-end latency with different densities.

(iii) Throughput: Figures 11 and 12 show that the proposed method has a better performance. We have obtained the throughput with different number of nodes at different times. However, the proposed model represents that at different times and vehicle densities, the proposed method has a better performance in comparison with the ClouDiV protocol. According to this fact that in the proposed model, the path detection processes, especially in the Reactive routing process, are carried out through a number of UAVs, so more requests are completed, which is better than ClouDiV protocol that needs to produce routing packets while delivering data packets, and requests require more time to be completed.

(iv) The number of lost packets: Figure 13 shows that the number of lost packets is reduced in the proposed method. Since in the proposed method the packets have a greater chance of reaching destinations, as sometimes, the unmanned aerial vehicles (UAVs) select a shorter distance and a reliable path to deliver 


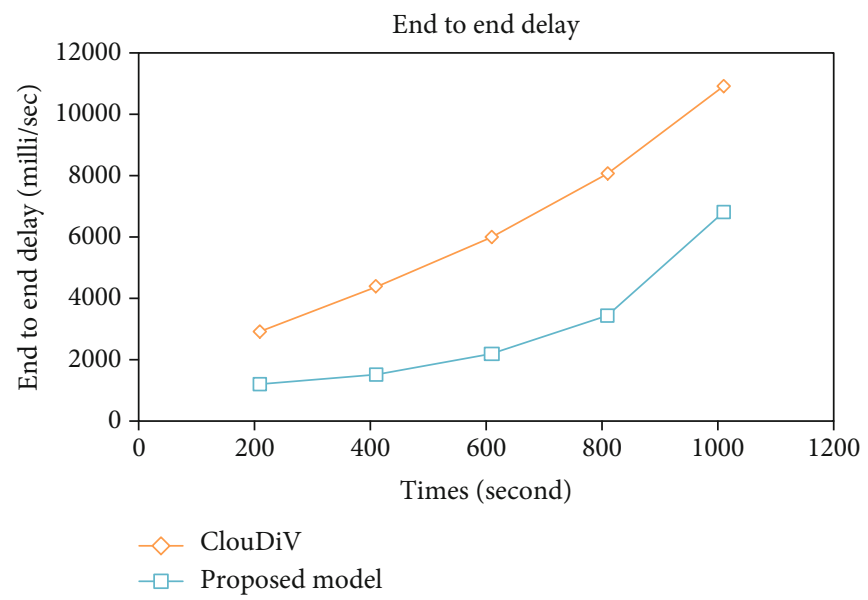

Figure 10: End-to-end latency at variable times.

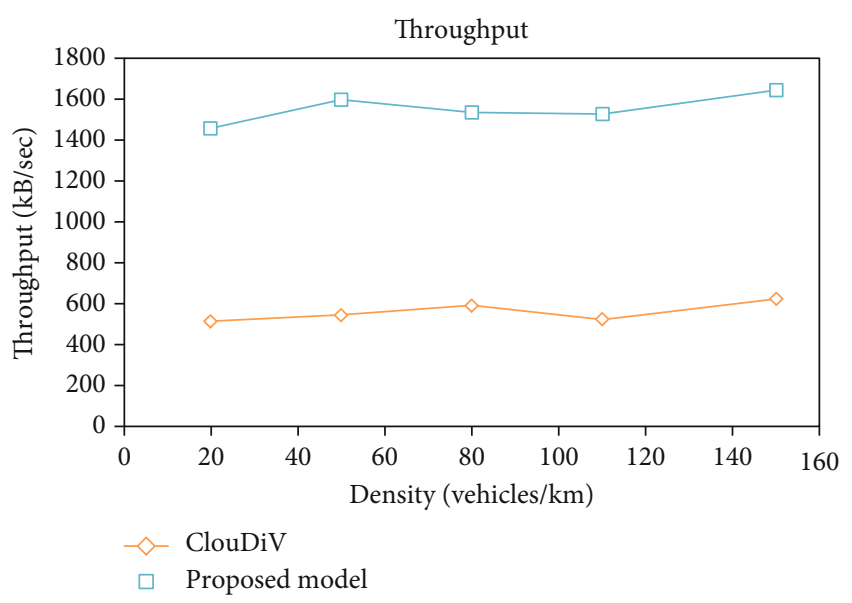

FIgURE 11: Throughput with different densities.

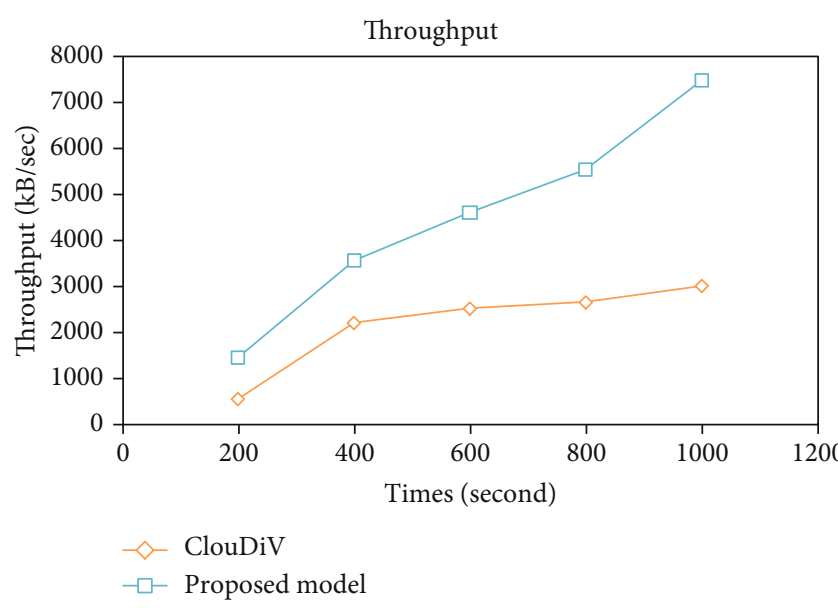

Figure 12: Throughput at variable times.

the packet to the destination, even when the network is dispersed or there is not any node in the neighborhood of the source, while in ClouDiV, it is possible that the requested path by the source node has not

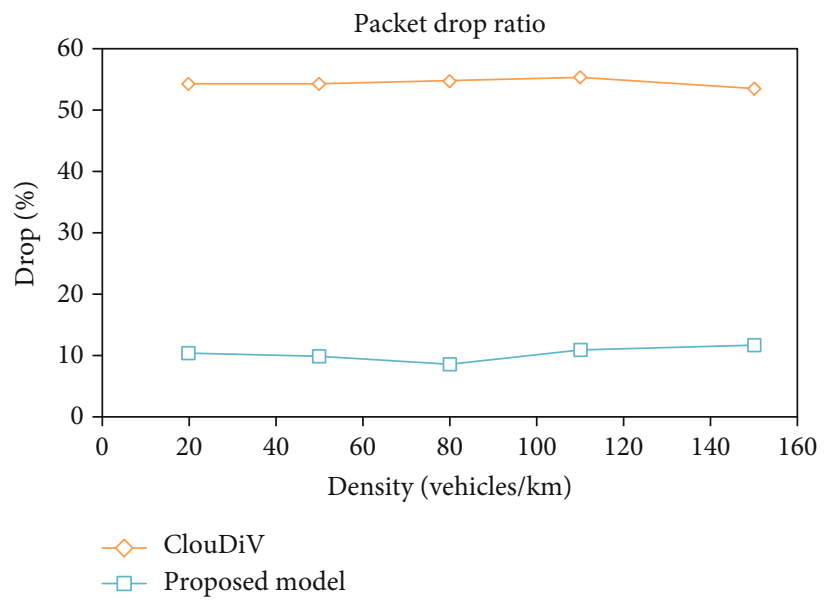

Figure 13: The number of lost packets with different densities.

yet been found in the path detection process, or the route to the destination has more hops, so it is more likely that the packet is lost

However, according to Figure 14, the proposed model has acceptable results at different times which is due to this fact that in ClouDiV protocol, when the communication path is broken at any time for any reason from the source to the destination, it is likely that the data packets are lost, while in the proposed method, by detecting the disconnected paths before data dissemination, the probability of losing data packets is reduced significantly and this implication reflects the better performance of the proposed model.

\section{Conclusion and Future Works}

In this paper, first, the connections in VANETs have been presented, and the use of cloud computing and UAVs in VANET communications has been given to deal with the problems of the vehicular network. Then, the proposed method has been proposed by modifying the ClouDiV protocol and, in fact, using the cloud computing infrastructure with utilization unmanned aerial vehicle (UAV) in urban 


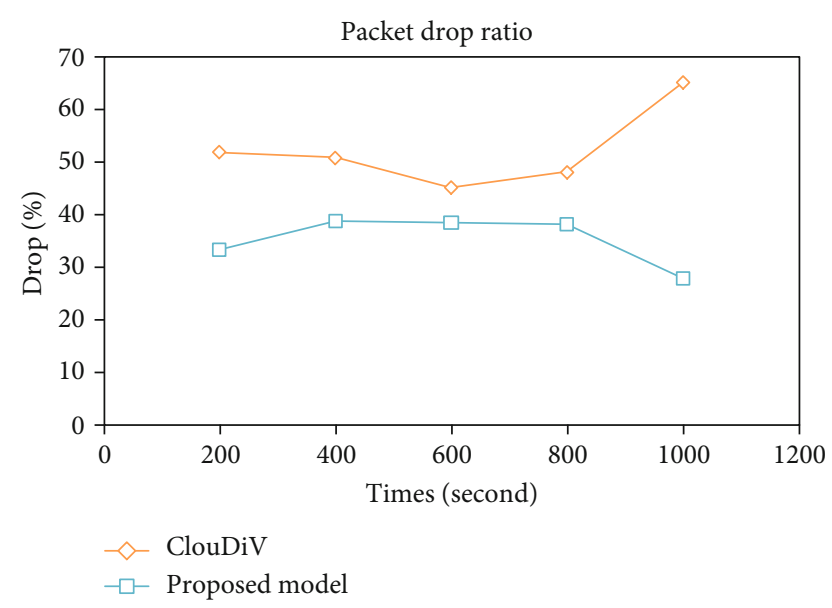

FIgURE 14: The number of lost packets at variable times.

areas. The simulation results show that the proposed method has better performance and efficiency in terms of improving packet delivery ratio, throughput, end-to-end latency, and the number of lost packets. However, UAVs can prevent the generation of additional routing packets, thus reducing network overhead. Also, in the proposed method, the UAVs will share connection information for VANET nodes on the earth and on data centers when there is no node in their neighborhood or for any reason they cannot communicate with each other. Therefore, the unmanned aerial vehicles (UAVs) will be able to be applied in the detection of broken paths.

The proposed method in this paper can be used to improve work in future researches on other geographic routing protocols in VANET networks or to be adapted to other environments such as highways and rural areas. The proposed method can also be improved on the security issue in VANET networks so that the broken connections can be better detected by examining the most important attacks on such network during routing.

\section{Data Availability}

This work is simulation.

\section{Conflicts of Interest}

We declare that we do not have any conflict of interest.

\section{References}

[1] M. Zarei, A. M. Rahmani, and H. Samimi, "Connectivity analysis for dynamic movement of vehicular ad hoc networks," Wireless Networks, vol. 23, no. 3, pp. 843-858, 2017.

[2] H. Akhtar and S. C. Sharma, "Performance Evaluation of Location-Based Geocast Routing using Directed Flooding Rectangular Forwarding Zone in City VANET," International Journal of Engineering and Technology Innovation, vol. 5, no. 4, pp. 264-278, 2015.

[3] S. Bitam and A. Mellouk, "Cloud Computing-Based Message Dissemination Protocol for Vehicular Ad Hoc Networks," in International Conference on Wired/Wireless Internet Commu- nication, M. Aguayo-Torres, J. Gómez, and A. Poncela, Eds., vol. 9071, pp. 32-45, Springer, Cham, 2015.

[4] Z. Zhang, G. Mao, and B. D. O. Anderson, "On the Information Propagation Process in Mobile Vehicular Ad Hoc Networks," IEEE Transactions on Vehicular Technology, vol. 60, no. 5, pp. 2314-2325, 2011.

[5] A. Agarwal, D. Starobinski, and T. D. C. Little, "Phase transition of message propagation speed in delay-tolerant vehicular networks," IEEE Transactions on Intelligent Transportation Systems, vol. 13, no. 1, pp. 249-263, 2012.

[6] Z. Zhang, G. Mao, and B. D. O. Anderson, "Stochastic characterization of information propagation process in vehicular ad hoc networks," IEEE Transactions on Intelligent Transportation Systems, vol. 15, no. 1, pp. 122-135, 2014.

[7] E. Baccelli, P. Jacquet, B. Mans, and G. Rodolakis, "Highway vehicular delay tolerant networks: information propagation speed properties," IEEE Transactions on Information Theory, vol. 58, no. 3, pp. 1743-1756, 2012.

[8] L. Cheng and S. Panichpapiboon, "Effects of intervehicle spacing distributions on connectivity of VANET: a case study from measured highway traffic," IEEE Communications Magazine, vol. 50, no. 10, pp. 90-97, 2012.

[9] S. Durrani, X. Zhou, and A. Chandra, "Effect of Vehicle Mobility on Connectivity of Vehicular Ad Hoc Networks," in 2010 IEEE 72nd Vehicular Technology Conference - Fall, pp. 1-5, Ottawa, ON, 2010.

[10] M. Khabazian and M. Ali, "A Performance Modeling of Connectivity in Vehicular Ad Hoc Networks," IEEE Transactions on Vehicular Technology, vol. 57, no. 4, pp. 2440-2450, 2008.

[11] A. Cardote, S. Sargento, and P. Steenkiste, "On the connection availability between relay nodes in a VANET," in 2010 IEEE Globecom Workshops, pp. 181-185, Miami, FL, 2010.

[12] R. Nagel, "The effect of vehicular distance distributions and mobility on VANET communications," in 2010 IEEE Intelligent Vehicles Symposium, pp. 1190-1194, San Diego, CA, 2010.

[13] J. Wu, "Connectivity of mobile linear networks with dynamic node population and delay constraint," IEEE Journal on Selected Areas in Communications, vol. 27, no. 7, pp. 12181225, 2009.

[14] P. Tomar, B. K. Chaurasia, and G. S. Tomar, "State of the art of data dissemination in VANETs," International Journal of Computer Theory an d Engineering, vol. 2, no. 6, pp. 957962, 2010.

[15] J. Karedal, N. Czink, A. Paier, F. Tufvesson, and A. F. Molisch, "Path loss modeling for vehicle-to-vehicle communications," IEEE Transactions on Vehicular Technology, vol. 60, no. 1, pp. 323-328, 2011.

[16] P. C. Neelakantan and A. V. Babu, "Connectivity Analysis of Vehicular Ad Hoc Networks from a Physical Layer Perspective," Wireless Personal Communications, vol. 71, no. 1, pp. 45-70, 2013.

[17] O. Onubogu, K. Ziri-Castro, D. Jayalath, K. Ansari, and H. Suzuki, "Empirical vehicle-to-vehicle pathloss modeling in highway, suburban and urban environments at $5.8 \mathrm{GHz}$," in 2014 8th International Conference on Signal Processing and Communication Systems (ICSPCS), pp. 1-6, Gold Coast, QLD, 2014.

[18] G. Yan and D. B. Rawat, "Vehicle-to-vehicle connectivity analysis for vehicular ad-hoc networks," Ad Hoc Networks, vol. 58, pp. 25-35, 2017. 
[19] Z. Amjad, W. Song, and K. Ahn, "Two-Level Hierarchical Routing Based on Road Connectivity in VANETs," in 2016 International Conference on Industrial Engineering, Management Science and Application (ICIMSA), pp. 1-5, Jeju, 2016.

[20] N. Alsharif and X. Shen, "\$i\$CAR-II: Infrastructure-Based Connectivity Aware Routing in Vehicular Networks," IEEE Transactions on Vehicular Technology, vol. 66, no. 5, pp. 4231-4244, 2017.

[21] M. Tavan, R. D. Yates, and D. Raychaudhuri, "Connected vehicles under information-centric architectures," in 2016 IEEE Vehicular Networking Conference (VNC), pp. 1-8, Columbus, OH, 2016.

[22] Q. Luo, C. Li, Q. Ye, T. H. Luan, L. Zhu, and X. Han, "CFT: A Cluster-based File Transfer Scheme for highway VANETs," in 2017 IEEE International Conference on Communications (ICC), pp. 1-6, Paris, 2017.

[23] Z. Li, Y. Song, and J. Bi, "CADD: connectivity-aware data dissemination using node forwarding capability estimation in partially connected VANETs," Wireless Networks, vol. 25, no. 1, pp. 379-398, 2019.

[24] H. Qin and C. Yu, "A road network connectivity aware routing protocol for Vehicular Ad Hoc Networks," in 2017 IEEE International Conference on Vehicular Electronics and Safety (ICVES), pp. 57-62, Vienna, 2017.

[25] S. Li, Z. Li, X. Ge, J. Zhang, and M. Jo, "Multi-hop links quality analysis of 5G enabled vehicular networks," in 2017 9th International Conference on Wireless Communications and Signal Processing (WCSP), pp. 1-6, Nanjing, 2017.

[26] X. Wang, D. Wang, and Q. Sun, "Reliable routing in IP-based VANET with network gaps," Computer Standards \& Interfaces, vol. 55, pp. 80-94, 2018.

[27] H. Fernandez, L. Rubio, V. M. Rodrigo-Penarrocha, and J. Reig, "Path Loss Characterization for Vehicular Communications at $700 \mathrm{MHz}$ and $5.9 \mathrm{GHz}$ Under LOS and NLOS Conditions," IEEE Antennas and Wireless Propagation Letters, vol. 13, pp. 931-934, 2014.

[28] P. Liu, D. W. Matolak, B. Ai, and R. Sun, "Path loss modeling for Vehicle-to-Vehicle communication on a slope," IEEE Transactions on Vehicular Technology, vol. 63, no. 6, pp. 2954-2958, 2014.

[29] S. Shelly and A. V. Babu, "Link residual lifetime-based next hop selection scheme for vehicular ad hoc networks," EURASIP Journal on Wireless Communications and Networking, vol. 2017, no. 1, 2017.

[30] M. Nabil, A. Hajami, and A. Haqiq, "A stable route prediction and the decision taking at sending a data packet in a highway environment," in Proceedings of the 2nd international Conference on Big Data, Cloud and Applications, pp. 1-6, Tetouan, Morocco, 2017.

[31] Y. Qin, D. Huang, and X. Zhang, "VehiCloud: Cloud Computing Facilitating Routing in Vehicular Networks," in 2012 IEEE 11th International Conference on Trust, Security and Privacy in Computing and Communications, pp. 1438-1445, Liverpool, 2012.

[32] Y.-W. Lin, J.-M. Shen, and H.-C. Weng, "Cloud-Supported Seamless Internet Access in Intelligent Transportation Systems," Wireless Personal Communications, vol. 72, no. 4, pp. 2081-2106, 2013.

[33] S. Olariu, T. Hristov, and G. Yan, "The Next Paradigm Shift: From Vehicular Networks to Vehicular Clouds," in Mobile Ad Hoc Networking: Cutting Edge Directions, S. Basagni, M.
Conti, S. Giordano, and I. Stojmenovic, Eds., John Wiley \& Sons, Inc, Hoboken, NJ, USA, Second edition, 2013.

[34] R. Hussain, J. Son, H. Eun, S. Kim, and H. Oh, "Rethinking Vehicular Communications: Merging VANET with cloud computing," in 4th IEEE International Conference on Cloud Computing Technology and Science Proceedings, pp. 606-609, Taipei, 2012.

[35] K. Mershad and H. Artail, "Finding a STAR in a Vehicular Cloud," IEEE Intelligent Transportation Systems Magazine, vol. 5, no. 2, pp. 55-68, 2013.

[36] D. Baby, R. D. Sabareesh, R. A. K. Saravanaguru, and A. Thangavelu, "VCR: Vehicular Cloud for Road Side Scenarios," in Advances in Computing and Information Technology, N. Meghanathan, D. Nagamalai, and N. Chaki, Eds., vol. 178 of Advances in Intelligent Systems and Computing, pp. 541552, Springer, Berlin, Heidelberg, 2013.

[37] M. Le, J. Park, and M. Gerla, "UAV Assisted Disruption Tolerant Routing," in MILCOM 2006 - 2006 IEEE Military Communications conference, pp. 1-5, Washington, DC, 2006.

[38] O. S. Oubbati, A. Lakas, N. Lagraa, and M. B. Yagoubi, "CRUV: Connectivity-based traffic density aware routing using UAVs for VANets," in 2015 International Conference on Connected Vehicles and Expo (ICCVE), pp. 68-73, Shenzhen, 2015.

[39] O. S. Oubbati, A. Lakas, F. Zhou, M. Güneş, N. Lagraa, and M. B. Yagoubi, "Intelligent UAV-assisted routing protocol for urban VANETs," Computer Communications, vol. 107, pp. 93-111, 2017.

[40] O. S. Oubbati, A. Lakas, N. Lagraa, and M. B. Yagoubi, "UVAR: An intersection UAV-assisted VANET routing protocol," in 2016 IEEE Wireless Communications and Networking Conference, pp. 1-6, Doha, 2016. 